\title{
THE INTERACTION OF ROTATION WITH CONVECTION
}

\author{
BERNARD R. DURNEY \\ High Altitude Observatory, National Center for Atmospheric Research*, Boulder, Colo., U.S.A.
}

\begin{abstract}
The equations for a rotating convective spherical shell are solved in the Herring approximation as an initial value problem. The main results are

(1) The most unstable modes (those that maximize the heat flux) correspond to convective cells stretching from pole to pole.

(2) The calculations of the Reynolds stresses show transport of angular momentum towards the equator. That is, differential rotation sets in with equatorial acceleration.

(3) The convective heat transport is maximum at the equator. This would give rise to an equatorpole flux difference.

(4) If convection is non-axisymmetric (as in the most unstable modes) there are no time independent solutions. The time dependence is oscillatory and of the form $\omega t+m \phi$.
\end{abstract}

\section{Theory}

In a system of coordinates rotating with an angular velocity $\Omega$, the basic equations can be written:

$$
\begin{aligned}
& \frac{1}{\sigma} \frac{\partial}{\partial t} \mathbf{\nabla} \times \mathbf{U}-\nabla \times \nabla^{2} \mathbf{U}=-\frac{1}{\sigma} \nabla \times(\mathbf{U} \cdot \nabla) \mathbf{U} \\
& +\mathscr{R}_{1} \nabla \times g(r) \mathbf{r} T+\mathscr{T}_{1}(\hat{\omega} \cdot \nabla) \mathbf{U} \\
& \operatorname{div} \mathbf{U}=0 \\
& \left(\frac{\partial}{\partial t}-\nabla^{2}\right) T=-\nabla \cdot(\mathbf{U} T) .
\end{aligned}
$$

In Equations (1) all quantities are dimensionless, $\sigma$ is the Prandtl number, $\mathscr{R}_{1}=$ $\mathscr{R} /(1-\eta)^{3}$ where $\mathscr{R}$ is the Rayleigh number and $\eta=0.8$ is the internal radius of the spherical shell in units of $R_{0}$, the external radius;

$$
\mathscr{T}_{1}=2 \Omega R_{0}^{2} / v=\mathscr{T}_{a}^{1 / 2} /(1-\eta)^{2}
$$

where $\mathscr{T}_{a}$ is the Taylor number; and $\hat{\omega}$ is a unit vector in the direction of the angular velocity. The unit of distance is $R_{0}$, and not, $d$, the thickness of the spherical shell, this explains the factors $(1-\eta)^{3}$ and $(1-\eta)^{2}$ in $\mathscr{R}_{1}$ and $\mathscr{T}_{1}$, respectively. The factor $g(r)$ takes into account the variation of gravity with radial distance.

We expand the velocity field in basic poloidal and toroidal vectors:

$$
\mathbf{U}=\sum_{L, m}\left[\mathbf{P}\left(p_{L}^{m}(r, t) Y_{L}^{m}(\theta, \varphi)\right)+\mathbf{T}\left(t_{L}^{m}(r, t) Y_{L}^{m}(\theta, \varphi)\right)\right]
$$

* The National Center for Atmospheric Research is sponsored by the National Science Foundation. 
In spherical coordinates the components of $\mathbf{P}\left(p_{L}^{m} Y_{L}^{m}\right)$ and $\mathbf{T}\left(t_{L}^{m} Y_{L}^{m}\right)$ are given by

$$
\begin{aligned}
P^{(r)} & =\frac{(L+1) L}{r^{2}} p_{L}^{m} Y_{L}^{m} \\
P^{(\theta)} & =\frac{1}{r} \frac{\partial p_{L}^{m}}{\partial r} \frac{\partial Y_{L}^{m}}{\partial \theta}, \\
P^{(\varphi)} & =\frac{1}{r \sin \theta} \frac{\partial p_{L}^{m} \partial Y_{L}^{m}}{\partial r} \partial \varphi
\end{aligned}
$$

and

$$
\begin{aligned}
T^{(r)} & =0 \\
T^{(\theta)} & =\frac{t_{L}^{m}}{r \sin \theta} \partial Y_{L}^{m} \\
T^{(\varphi)} & =-\frac{t_{L}^{m} \partial Y_{L}^{m}}{r} \partial \theta
\end{aligned}
$$

The poloidal and toroidal vectors defined by (3) form a complete orthogonal set for solenoidal vector fields.

For the temperature we take

$$
T=\frac{1}{1-\eta}\left(\frac{\eta}{r}-1\right)+\psi(r, t)+\Theta(\mathbf{r}, t)
$$

with

$$
\Theta(\mathbf{r}, t)=\sum_{L, m} \Theta_{L}^{m}(r, t) Y_{L}^{m}(\theta, \varphi)
$$

We take the spherical harmonics appearing in (2) and (3) as defined by Condon and Shortley (1951).

In Herring's approximation $(1963,1964)$ the fluctuating self interactions, that is, the terms $(1 / \sigma)(\mathbf{U} \cdot \nabla \mathbf{U}-\overline{U \cdot \nabla \mathbf{U}})$ and $(U \cdot \nabla \Theta-\overline{U \cdot \nabla} \Theta)$ are neglected. The main effect of the small scale part of these terms is to give rise to a turbulent viscosity and conductivity; the Rayleigh and Taylor number should then be defined in terms of these last quantities.

The equations for $\Theta_{L}^{m}(r, t)$ and $\psi(r, t)$ (the fluctuating and average parts of the distortion in temperature from its purely conductive value) and the equations for the poloidal and toroidal components of the velocity field are found to be:

$$
\begin{aligned}
& \left(\frac{\partial}{\partial t}-\mathscr{D}_{L}-\frac{(L+1) L}{r^{2}}\right) \psi=-\frac{1}{4 \pi r^{2}} \sum_{L, m}^{|m| \leqslant L}(L+1) L \frac{\partial}{\partial r}\left(r P_{L}^{m} \Theta_{L}^{* m}\right), \\
& \left(\begin{array}{c}
\partial \\
\partial t
\end{array}-\mathscr{D}_{L}\right) \Theta_{L}^{m}=\frac{(L+1) L}{r} P_{L}^{m}\left[\frac{\eta}{(1-\eta) r^{2}}-\frac{\partial \psi}{\partial r}\right],
\end{aligned}
$$




$$
\begin{aligned}
& \frac{1}{\sigma} \frac{\partial}{\partial t} \mathscr{D}_{L} P_{L}^{m}-\frac{i m \mathscr{T}_{1}}{(L+1) L} \mathscr{D}_{L} P_{L}^{m}-\mathscr{D}_{L}^{2} P_{L}^{m} \\
&=-\mathscr{R}_{1} g(r) \Theta_{L}^{m}+\mathscr{T}_{1}\left\{A(L, m)(L-1)(L-2) T_{L-1}^{m} / L\right. \\
&-A(L+1, m)(L+2)(L+3) T_{L+1}^{m} /(L+1) \\
&\left.-A(L, m)(L-1) r T_{L-1}^{\prime m} / L-A(L+1, m)(L+2) r T_{L+1}^{\prime m} /(L+1)\right\}
\end{aligned}
$$

$$
\begin{aligned}
\frac{1}{\sigma} \frac{\partial}{\partial t} T_{L}^{m}- & \frac{i m \mathscr{T}_{1}}{(L+1) L} T_{L}^{m}-D_{L} T_{L}^{m} \\
= & -\mathscr{T}_{1}\left\{A(L, m)(L-1)^{2} P_{L-1}^{m} / L r^{2}-A(L+1, m)\right. \\
& \times(L+2)^{2} P_{L+1}^{m} /(L+1) r^{2}-A(L, m)(L-1) P_{L-1}^{\prime m} / r L \\
& \left.-A(L+1, m)(L+2) P_{L+1}^{\prime m} / r(L+1)\right\}
\end{aligned}
$$

where

$$
\begin{aligned}
& \mathscr{D}_{L}=\frac{\mathrm{d}^{2}}{\mathrm{~d} r^{2}}+\frac{2}{r} \frac{\mathrm{d}}{\mathrm{d} r}-\frac{(L+1) L}{r^{2}} \\
& D_{L}=\frac{\mathrm{d}^{2}}{\mathrm{~d} r^{2}}+\frac{4 \mathrm{~d}}{r} \frac{2-(L+1) L}{r^{2} r}+\frac{2-}{\mathrm{d}} \\
& P_{L}^{m}=p_{L}^{m} / r ; \quad T_{L}^{m}=t_{L}^{m} / r^{2} ; \quad T_{L}^{\prime m}=\frac{\mathrm{d} T_{L}^{m}}{\mathrm{~d} r} ; \quad P_{L}^{\prime m}=\frac{\mathrm{d} P_{L}^{m}}{\mathrm{~d} r} \\
& A(L, m)=[(L+m)(L-m) /(2 L+1)(2 L-1)]^{1 / 2} .
\end{aligned}
$$

Equations (4) are the fundamental equations of the problem. We assume free surface boundary conditions at $r=\eta, 1$. It is easily seen that they imply $P_{L}^{m}=P_{L}^{\prime \prime m}=T_{L}^{\prime m}=0$ at $r=\eta, 1$. The boundary conditions for the temperature are as usual $\Theta=\psi=0$ at $r=\eta, 1$.

We neglect the time derivatives in Equations (4c) and (4d). In the absence of rotation modes with different $L$ 's do not interact. The integration in time of Equations (4) shows then that after a sufficiently long time only the mode with $L=10$ remains different from zero (Durney, 1968a). Another value of $L$ for the non-zero mode would have been found for a different thickness of the spherical shell (in the present case, the thickness is $0.2 R_{0}$ ).

Rotation couples, through the Taylor number, modes with different $L$ 's. For $m=0$, there exist time-independent solutions (Durney, 1968b). For $m \neq 0$ no time-independent solutions were found. This was attributed to the fact for $m \neq 0$ the convective modes of polytropes show overstability (Durney and Skumanich, 1968). It was then decided to integrate in time Equations (4) for a given value of $m$ by keeping only the $L=8,10,12$ modes for $P_{L}^{m}$ and $\Theta_{L}^{m}$ and the $L=9,11$ modes for $T_{L}^{m}$. This should be a good approximation for small Taylor numbers.

To perform the time integration $P_{L}^{m}, \Theta_{L}^{m}, \psi(r, \mathrm{t})$ and $T_{L}^{m}$ were expanded in terms of a complete set of functions of $r$ satisfying the boundary conditions. The coefficients in these expansions are time-dependent, and from Equations (4) it is possible to obtain ordinary differential equations for these coefficients. 


\section{Numerical Results}

The values of the Rayleigh number and Taylor number were chosen to be equal to 1500 and 4 , respectively.

A. THE MOST UNSTABLE MODE

Table I gives the value of the heat flux as function of $m$.

TABLE I

\begin{tabular}{llll}
\hline Heat Flux $\times 10^{-2}$ & 0.99919 & 0.99924 & 0.99941 \\
$m$ & 0 & 1 & 2 \\
0.99968 & 1.00007 & 1.00056 & \\
3 & 4 & 5 & \\
1.00116 & 1.00188 & 1.0027 & \\
6 & 7 & 8 & \\
1.00357 & 1.00459 & & \\
9 & 10 & & \\
\hline
\end{tabular}

The most unstable mode is thus the one with $m=10$. This is in agreement with Busse's (1969) results who finds that the Rayleigh number for the onset of convection is a minimum for $m=L$.

\section{B. DIFFERENTIAL ROTATION}

The average of the azimuthal velocity $U_{\varphi}$ over $\varphi$ is zero. Differential rotation, as observed in the sun, should, however, be a property of a rotating convective spherical shell. Once the velocities are known in the Herring approximation, it is possible to evaluate the torque $T(\theta) d \theta$, exerted by the Reynolds stresses on an annulus of thickness $d$ (the thickness of the spherical shell). The polar angle of the annulus is $\theta$. This torque is given in Figure 1 and shows equatorial acceleration.

\section{EQUATOR-POLE FLUX DIFFERENCE}

Figure 2 shows the convective flux $\overline{U_{r} \Theta}$ (the average is over $\varphi$ ) as function of $\theta$ for $r=0.9$ which is the surface halfway between the inner $(r=0.8)$ and outer $(r=1)$ surfaces. The convective flux is larger at the equator. This could give rise to an equator-pole flux difference as observed by Dicke and Goldenberg (1967). A possible interpretation of this effect is based on the interaction of rotation and convection (Roxburgh, 1967a, b, 1969; Durney and Roxburgh, 1969).

\section{GRAVITATIONAL-GYROSCOPIC WAVES}

The velocity field $\mathbf{U}$ and the fluctuation in temperature $\Theta$ are time-dependent. The 


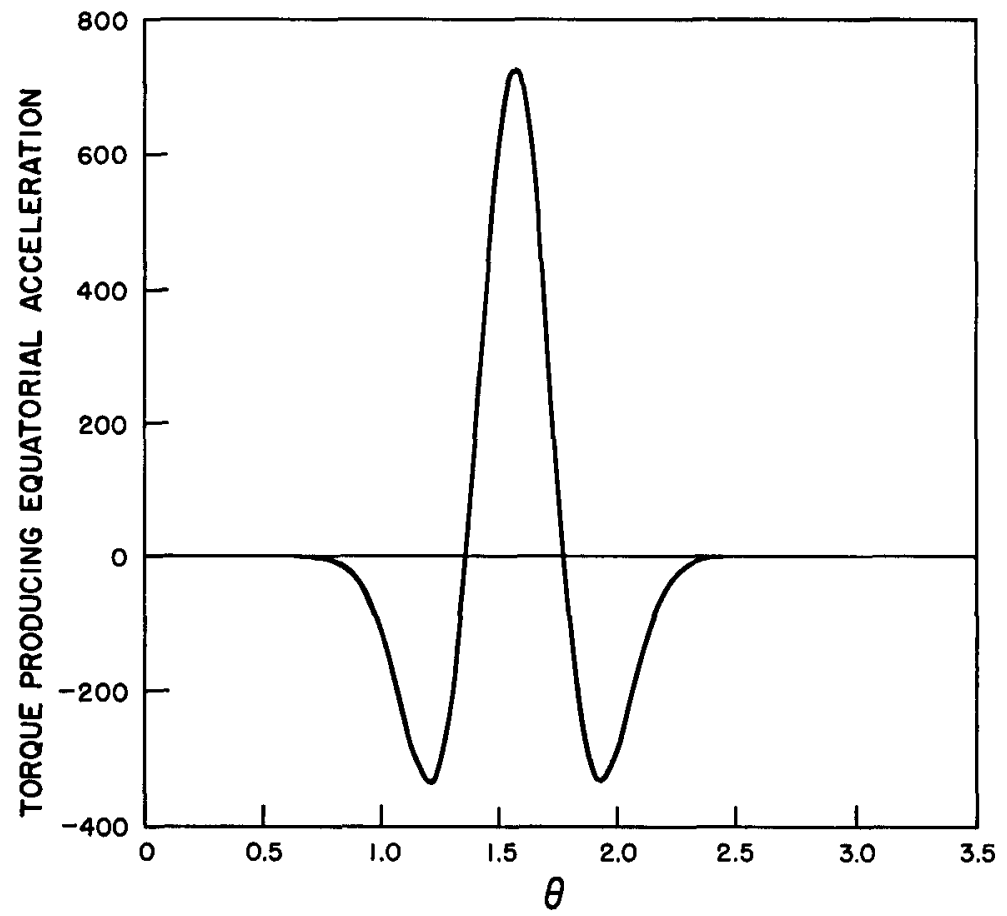

Fig. 1. Torque exerted by the Reynolds stresses on an annulus of polar angle $\theta$. This torque produces differential rotation.

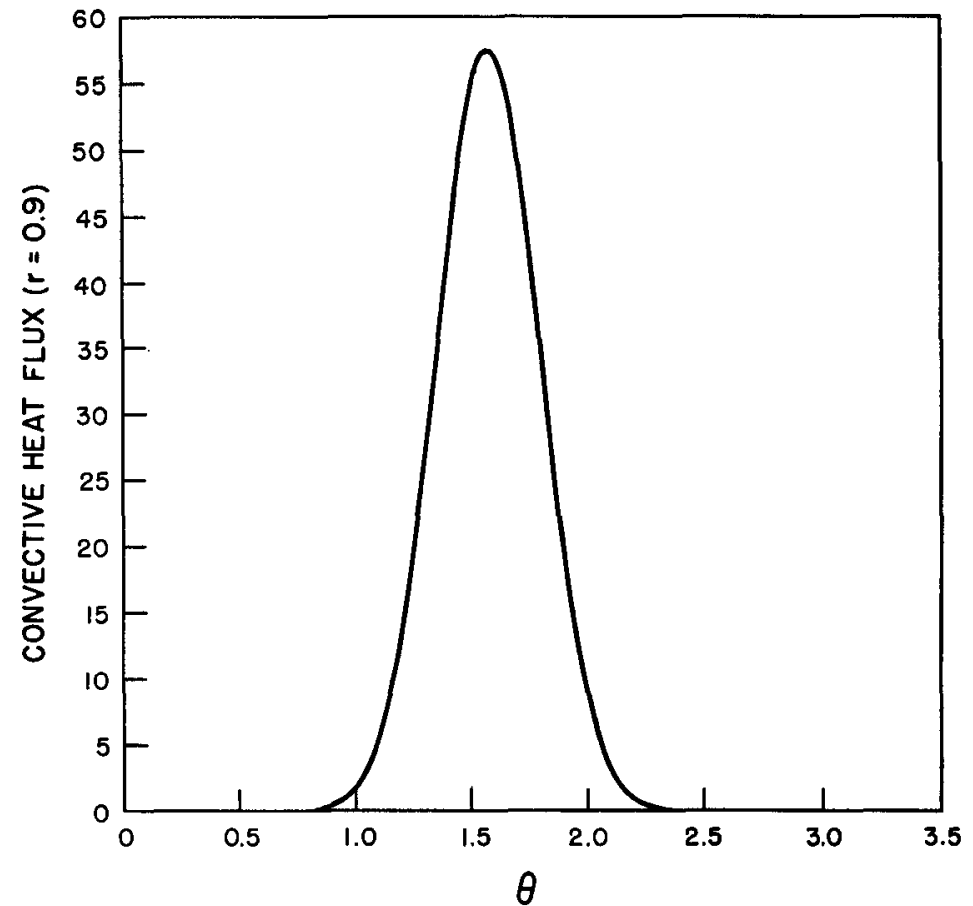

Fig. 2. Convective heat flux at a surface halfway between the inner and outer surfaces. 
torque and the convective heat flux as given by Figures 1 and 2 are nevertheless timeindependent. More generally if $A$ and $B$ stand for any of the quantities $U$ and $\Theta$ then the average of $A B$ over $\varphi \overline{(A B)}$ is constant in time for sufficiently large values of the time. As $A$ or $B$ are of the form $A=A_{1}(r, \theta, t) \cos m \varphi+A_{2}(r, \theta, t) \sin m \varphi$ the constancy of $\overline{A B}$ suggests the following time-dependence:

$$
A=A_{1}(r, \theta) \cos (\omega t+m \varphi)+A_{2}(r, \theta) \sin (\omega t+m \varphi)
$$

The value of $\omega$ was found to be equal to $\omega=3.153$.

This time dependence is identical to that of the states of marginal stability of the convective modes of a rotating polytrope (Durney and Skumanich, 1968). These 'gravitational-gyroscopic' waves have some similarities with Rossby waves as introduced by Ward (1965, 1966), Starr and Gilman (1965) and Gilman (1967) to explain the differential rotation of the sun. If giant convective cells exist in the sun, the use of the Herring approximation can be justified to explain qualitatively certain features of the convective zone of the sun. On theoretical grounds Simon and Weiss (1969) have suggested the existence of giant convective cells and Howard (1969) has observed upward and downward motions extending from pole to pole.

\section{Acknowledgements}

The author is grateful to Professors P. Gilman and I. Roxburgh and to Dr. A. Skumanich for many illuminating discussions.

\section{References}

Busse, F. H.: 1969, Max Planck Institute Report MPI-PAE/Astro 15.

Condon, E. U. and Shortley, G. H.: 1951, The Theory of Atomic Spectra, Cambridge University Press, p. 52.

Dicke, R. H. and Goldenberg, H. M.: 1967, Nature 214, 1294.

Durney, B. R.: 1968a, J. Atmos. Sci. 25, 372.

Durney, B. R.: 1968b, J. Atmos. Sci. 25, 771.

Durney, B. R. and Roxburgh, I. W.: 1969, Nature 221, 646.

Durney, B. R. and Skumanich, A.: 1968, Astrophys. J. 152, 225.

Gilman, P. A.: 1967, Part I, II, III, J. Atmos. Sci. 24, 101.

Herring, J. R.: 1963, J. Atmos. Sci. 20, 325.

Herring, J. R.: 1964, J. Atmos. Sci. 21, 277.

Howard, R.: 1969, unpublished.

Roxburgh, I. W.: 1967a, Nature 213, 1077.

Roxburgh, I. W.: 1967b, Nature 216, 1286.

Roxburgh, I. W.: 1969, The Application of Modern Physics to the Earth and Planetary Interiors. Wiley London.

Simon, G. W. and Weiss, N. O.: 1969, Z. Astrophys. 69, 435.

Starr, V. P. and Gilman, P. A.: 1965, Astrophys. J. 141, 1119.

Ward, F.: 1965, Astrophys. J. 141, 534.

Ward, F.: 1966, Astrophys. J. 145, 416. 


\section{Discussion}

Roxburgh: It is important to emphasize that giant cells have been observed by Howard and that these have the same banana-mode shape.

Ruben: Generally a rotating star should be hotter at the pole than at the equator. Does your effect of mainly equatorial heat transport compensate this?

Durney: The effect of rotation on convection should be much larger than the von Zeipel effect.

Fricke: I would mention that Fritz Busse in a recent paper concerning the equatorial acceleration of the sun came to similar results with such an analysis.

Durney: Yes. Busse's treatment is based on a double expansion: one, in the convective amplitudes and the other in the Taylor number. 